\title{
Low sleep and diet quality impact on well-being among Mexican college students
}

\author{
Maria Fernanda Zeron-Rugerio ${ }^{1,2}$, Ana Eugenia Ortega-Regules ${ }^{3}$, \\ Maria Armida Patricia Porras-Loaiza ${ }^{3}$, Giovana Longo-Silva ${ }^{1,4}$, Trinitat Cambras ${ }^{5}$ and \\ Maria Izquierdo-Pulido ${ }^{1,2}$ \\ ${ }^{1}$ Department of Nutrition, Food Science and Gastronomy. School of Pharmacy and Food Science. University of \\ Barcelona, Barcelona, Spain, \\ ${ }^{2}$ Institut de Recerca en Nutrició i Seguretat Alimentària (INSA-UB) University of Barcelona, Barcelona, Spain, \\ ${ }^{3}$ Department of Health Sciences, School of Science, Universidad de las Americas Puebla, Puebla, Mexico, \\ ${ }^{4}$ Nutrition Faculty. Federal University of Alagoas, Alagoas, Brazil and \\ ${ }^{5}$ Department of Biochemistry and Physiology. School of Pharmacy and Food Science. University of Barcelona, \\ Barcelona, Spain
}

\begin{abstract}
Introduction

Understanding health behaviors, such as sleep and dietary patterns, is important for health promotion and disease prevention. College period is a complicated, difficult time to maintain healthy behaviors. In this regard, little is known about the impact of sleep and diet quality on well-being among college young adults. Hence, we aimed to study the impact of sleep and diet quality on well-being in college students.
\end{abstract}

\section{Materials and Methods}

Three hundred-twelve Mexican college students (82\% females; $20.6 \pm 3.4 y e a r s)$ were included in a cross-sectional study. Participants completed the following validated questionnaires: Well-being Index to assess well-being (high scores indicate high well-being), Pittsburg Sleep Quality Index for sleep quality (scores $>5$ indicate poor sleep quality), and a validate version of the Health Eating Index for Mexican population to assess diet quality (scores $>80$ indicate good diet quality). BMI and physical activity were also assessed. Scores were treated as continuous variables, and statistical analysis were performed using SPSS v24 and adjusted for confounding variables.

\section{Results}

Sixty-seven percent of our population showed a poor sleep quality and eighty-four percent needs to make changes in their diet in order to improve its quality. On the other hand, fifty-one percent of the studied population had a low well-being perception. Poor sleep quality $(\mathrm{p}<0.001)$ and low diet quality $(\mathrm{p}<0.001)$ associated with a lower well-being perception. In addition, poor sleep quality is associated with low diet quality $(p<0.05)$. Regarding food consumption, higher sweets $(p<0.001)$ and soft-drink $(p<0.001)$ intake was inversely associated with well-being scores. Likewise, high consumption of sweets $(\mathrm{p}<0.05)$ and soft-drink $(\mathrm{p}<0.05)$, and low fruit $(\mathrm{p}<0.05)$ and cereal $(\mathrm{p}<0.05)$ consumption are linked to poor sleep quality.

\section{Discussion}

Our results show that half of the studied population had a low well-being, and also that poor sleep and diet quality are frequent among college students. Interestingly, the fact that poor sleep and diet quality were associated to a lower perception of well-being highlights the importance of promoting sleep hygiene and diet quality among college students as an important strategy to improve well-being and health, even in young adults. In addition, we found that higher sweets and soft-drink consumption were associated to both low well-being perception and poor sleep quality. Hence, it is important to promote healthy habits regarding eating and sleeping well, in order to improve well-being perception among college students.

\section{Conflict of Interest}

There is no conflict of interest 